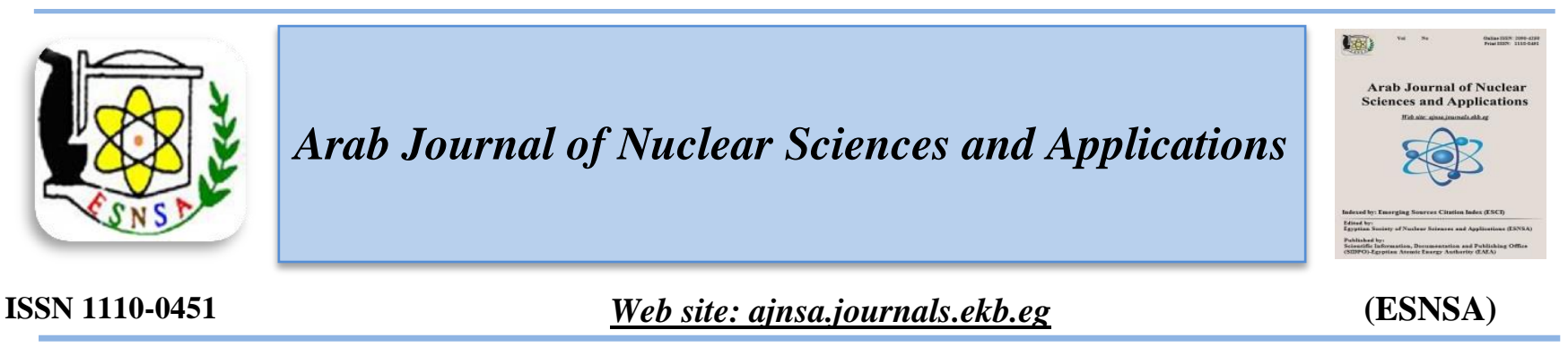

\title{
Dark Matter production in Association with Dilepton Resonance from Heavy Z' Decay Using Monte Carlo Simulation
}

\author{
S. Elgammal ${ }^{2}$, M. Abdelhakim ${ }^{1}{ }^{*}$, A.Y. Ellithi ${ }^{1}$ and M.T. Hussein ${ }^{1}$ \\ ${ }^{1}$ Physics Department, Faculty of Science, Cairo University, Cairo, Egypt \\ ${ }^{2}$ Center for theoretical physics, British University in Egypt, Elsherouk, Egypt
}

Received 18 May 2020 In the present work, the possible production of Dark Matter candidates in association with the Z' heavy Accepted 29 Nov. 2020 gauge boson was investigated. This study is performed by simulating events with large missing transverse momentum produced in proton-proton collisions at the Large Hadron Collider, at $13 \mathrm{TeV}$ center-of-mass energy and integrated luminosity of $136.2 \mathbf{f b}^{-1}$ corresponding to RUN II. The $Z$ ' boson is considered to decay leptonically. Three models beyond standard model were studied, which assume that the $Z^{\prime}$ is coupled to new dark sector states. The significances of the three models were tested. Keywords: Dark Matter, Z’ heavy gauge boson, Monte Carlo simulation, Large Hadron Collider

\section{Introduction}

Many cosmological evidences, supported by the recent observations [1], confirm the existence of an unknown form of matter named the Dark Matter (DM), and that it occupies approximately $27 \%$ of the total energy distribution in the Universe. Various types of candidates were suggested to compose DM [2]. One of the accepted hypotheses, which is in an agreement with the observed density, is that the bulk of DM has the form of electrically-neutral, nonbaryonic, nondecaying, and weakly-interacting massive particles (WIMPs) [3]. The Standard Model of particle physics (SM) [4] gave a satisfactory demonstration of the wellknown baryonic matter, but it does not introduce a suitable candidate for the DM. The DM and other several motivations lead to construct new models beyond SM (BSM) [5]. The masses of the DM candidate supposed are in the range between few $\mathrm{GeV}$ and few $\mathrm{TeV}$ [6], making it possible to be produced at the particle colliders, such as the Large Hadron Collider (LHC).
The searches for DM at the LHC are generally based on the production of a SM visible particle X, accompanied by large missing transverse momentum ptmiss from the invisible DM particles which escape the detector without leaving any track. The experimental signature of the search is known as: "mono - X". A host of mono - X searches have been carried out by the ATLAS and CMS collaborations looking for DM, but none of these searches marked a significant deviation from the SM [7-11]. In this study, a search for DM in the context of new models is considered, in which DM particles are produced in association with a $Z^{\prime}$ boson. The Z' new heavy boson is postulated to be massive, electrically-neutral, short-lived, and color-singlet [12]. The experimental signature we are looking for will be known as mono - Z' [13].

$A$ review is made for the three models of DM production accompanied by Z' boson, which are: Dark Higgs model (DHM), Light Vector model (LVM), and the light $Z$ ' with inelastic effective field theory (EFT) model [13]. The search is based on Monte Carlo (MC) simulation of the signals

Corresponding author: maha.abdelhakim@ @ern.ch

DOI: 10.21608/ajnsa.2020.30257.1353

CScientific Information, Documentation and Publishing Office (SIDPO)-EAEA 
evaluated from the three models in addition to the simulation of the SM backgrounds from protonproton collision at center-of-mass energy $\sqrt{\mathrm{S}}_{\mathrm{s}}=13$ $\mathrm{TeV}$ and integrated luminosity $L=136.2 \mathrm{fb}^{-1}$, which corresponds to the LHC certified RUN II, at the generator level. In the three models, Z' couples to new dark sector states which are considered as candidates of the DM. The Z' boson can decay leptonically into two opposite-sign charged leptons or hadronically into two jets. The leptonic decay is considered giving a rise to the resonance of $l^{+} l^{-},(l$ $=e, \mu)$ around $M_{Z}$. We are looking for final states with $l^{+} l^{-}+p_{t}^{\text {miss. }}$. In the following sections, the theoretical models and their parameters are discussed. Then the simulation techniques are displayed in addition to the events generation. Afterwards, the selection cuts and the strategy of the analysis are explained. Finally, the results and the significances are discussed.

\section{Mono-Z' models}

The DHM assumes the existence of a new massive scalar, called the dark-Higgs $h_{D}$, that couples to the $Z$ '. The new dark-Higgs scalar $h_{D}$ is produced at a process called dark-Higgsstrahlung, similar to the SM Higgs boson radiation from the $\mathrm{Z}$ boson [13]. If the dark-Higgs $h_{D}$ couples to new dark states, the observed signature will be $p_{t}^{\text {miss }}$, as shown from the Feynman diagram presented in Figure( 1a). In the second model, LVM, Z' can be radiated off-shell from the decay of dark sector states, as shown in Figure $(1 \mathrm{~b})$. The third model, inelastic EFT, considers two dark sector states $\chi_{1}$ and $\chi_{2}$ with an off-diagonal coupling to the Z' which is then very weakly coupled to fermions as shown in Figure $(1 \mathrm{c})$. The cross section of these models will depend on certain free parameters. For the DHM, the cross section depends on the mass of the dark Higgs and the mass of Z'. For the LVM and the inelastic EFT, the cross section depends on the mass of $Z^{\prime}$ and the mass of the dark matter candidate $\chi_{1}$ [13]. The values of the coupling constants used are equal unity $\left(\mathrm{g}_{\mathrm{x}}=\mathrm{g}_{\mathrm{hD}}=1\right)$ [13].

\section{Event Generation}

The signal samples, for mono $-Z$ ' models, are generated by Madgraph [14] and the hadronization by Pythia 8 [15]. For the DHM, the invariant mass $\mathrm{M}_{\mathrm{Z}}$, is taken to be $400 \mathrm{GeV}$, and the mass of the dark-Higgs scalar $h_{D}$ is chosen to be equal to the mass of the SM Higgs scalar which is $125 \mathrm{GeV}$. For the LVM, the mass of the heavy dark sector state $\chi_{2}$ is $800 \mathrm{GeV}$, that of $\mathrm{M}_{\mathrm{Z}}$, is $400 \mathrm{GeV}$, and the mass of the coupled light dark sector state $\chi_{1}$ is 200 $\mathrm{GeV}$. For the inelastic EFT model, $\mathrm{M}_{\mathrm{Z}}$ is $400 \mathrm{GeV}$, the mass of the two dark sector states are 50 and $200 \mathrm{GeV}$, respectively. Those values were chosen to suppress the non-peaking backgrounds around the Z' window. Table (1) shows the cross sections calculated for these three models considered in the search. the authors consider all the SM processes that constitute a dilepton $l^{+} l^{-}+p_{t}^{\text {miss }}$ final state as backgrounds for this search, which are listed in Table (2). These backgrounds include:

- Z+ jets generated by Madgraph and Pythia 8

- Diboson events: (WW,ZZ generated by Powheg and Pythia 8) and (WZ generated by Madgraph and Pythia8).

- Triboson events: (WWZ,WZZ and ZZZ generated by Pythia 8).

- top-quark events: $\mathrm{ttW}, \mathrm{ttZ}, \mathrm{tW}, \overline{\mathrm{tW}}$ and $\mathrm{tt}$ generated by Madgraph and Pythia 8 .

The cross sections are calculated using the following formula:

$$
\sigma=\mathrm{N} /(\varepsilon \mathrm{AL})
$$

where $\mathrm{N}$ is the number of events, $\mathrm{A}$ is the acceptance, and $\mathrm{L}$ is the total integrated luminosity.

(a)

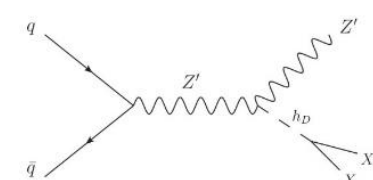

(b)

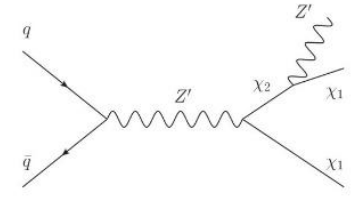

(c)

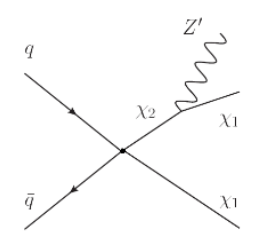

Fig. (1): Feynman diagrams are of the three mono $-Z$ ' models at the tree level. (a) diagram presents the associated production of the $Z$ ' and the dark-Higgs $h_{D}$ which couples to two new dark states, while the (b) diagram reveals the production of the $\chi_{1} \chi_{2}$, and the decay of dark sector state $\chi_{2}$ to $Z^{\prime}+\chi_{1}$ and $\chi_{1}$ is a possible dark matter candidate. The (c) diagram demonstrates the production of the $\chi_{1} \chi_{2}$, from the direct quark-anti quark annihilation, then the dark sector state $\chi_{2}$ decays to $Z^{\prime}+\chi_{1}$ and $\chi_{1}$ is a possible dark matter candidate [13].

Arab J. Nucl. Sci. \& Applic. Vol. 54, No.1 (2021) 
Table |(1): Signal samples and corresponding cross sections

\begin{tabular}{cc}
\hline Signal model & $\sigma(\mathrm{pb})$ \\
\hline DHM & $5.1970 \times 10^{-3}$ \\
\hline LVM & $0.0022 \times 10^{-3}$ \\
\hline EFT & $0.0024 \times 10^{-3}$ \\
\hline
\end{tabular}

Table (2): Background samples and corresponding cross sections

\begin{tabular}{cc}
\hline Background & $\sigma(\mathrm{pb})$ \\
\hline $\mathrm{tW}$ & 19.47 \\
\hline$\overline{\mathrm{tW}}$ & 19.47 \\
\hline $\mathrm{t} \overline{\mathrm{t}}$ & 87.31 \\
\hline $\mathrm{WW}$ & 118.7 \\
\hline $\mathrm{WZ}$ & 47.13 \\
\hline $\mathrm{ZZ}$ & 16.52 \\
\hline $\mathrm{ZZ} \rightarrow 4 l$ & 1.260 \\
\hline $\mathrm{ttW}$ & 0.200 \\
\hline $\mathrm{ttZ}$ & 0.250 \\
\hline $\mathrm{WWZ}$ & 0.170 \\
\hline $\mathrm{WZZ}$ & 0.050 \\
\hline $\mathrm{ZZZ}$ & 0.010 \\
\hline $\mathrm{Z}+\mathrm{jets}$ & 5765 \\
\hline
\end{tabular}

After these Monte Carlo background samples were generated, they are normalized to the LHC RUN II, at which $\sqrt{\mathrm{s}}_{\mathrm{s}}=13 \mathrm{TeV}$ and $L=136.2 \mathrm{f} \mathrm{b}^{-1}$, at the generator level.

\section{Event Selection and Analysis Strategy}

The heavy Z' boson leptonically decays to a pair of opposite-sign charged leptons (two electrons $\mathrm{e}^{+} \mathrm{e}^{-}$ or two muons $\mu^{+} \mu^{-}$), with high transverse momentum $p_{t}^{l}$. The selections for the analysis is designed to reconstruct a final state with this two leptons in association with $p_{t}^{\text {miss }}$ accounting for the DM candidate. The selection is made in the form of cuts applied on different kinematic parameters. The preliminary selection has the following requirements:

- $p_{t}^{l}>35 \mathrm{GeV}$

- $\mathrm{M}_{\mathrm{Z}}>70 \mathrm{GeV}$

- $\left|\eta_{l}\right|<2.5$

While $\mathrm{M}_{\mathrm{Z}}$ is the dilepton invariant mass, $\mathrm{p}_{\mathrm{t}}{ }_{\mathrm{t}}^{1}$ is the transverse momentum of the lepton, and $\left|\eta_{l}\right|$ is the absolute of the lepton pseudo-rapidity. The dilepton invariant mass was reconstructed, after the preliminary selection; which is shown in Figure (2 a) for the dielectron channel, Figure (2 b) displays the dimuon channel, and the combined dilepton channel is shown in Figure (3). Signals and backgrounds are normalized to respective cross sections, which are listed in Tables (1 and 2), respectively. The signals; DHM (Blue solid line), LVM (Green dotted line), and EFT model (Black dashed line) are observed to be embedded inside the SM backgrounds. However, a peak around 400 $\mathrm{GeV}$ representing the three signals. In order to effectively reduce the SM backgrounds and well maintain the signal events as possible, a set of further cuts was needed. The cut on the invariant mass of the dilepton is $\left(360<\mathrm{M}_{\underline{Z}}<425\right) \mathrm{GeV}$, in addition, a cut on $p_{t}^{\text {miss }}$ to be greater than $10 \mathrm{GeV}$ are applied in order to reduce the $\mathrm{Z}+$ jets and $\mathrm{tt}$ backgrounds. The final selection conditions are summarized in Table (3).

The efficiency of the final selection is calculated by dividing the number of events remaining after the final selection over the number of events remaining after the preliminary selection, and is shown in Figure (4). The final selection is highly efficient for signals and strongly suppresses the backgrounds.

\section{Results and Discussion}

After setting the baseline cuts to the MC samples, a comparison between signal and background was conducted in the $p_{t}^{\text {miss }}$ distributions. The $p_{t}^{\text {miss }}$ distributions, after the preliminary selection, are shown in Figure (5 a) for the dielectron, Figure (5 b) for the dimuon, and Figure ( 6 ) for the dilepton; for backgrounds (presented by colored histograms), and the signals; DHM (Blue solid line), LVM (Green dotted line), and EFT model (Black dashed line). It is observed that the highest background contribution is coming from $\mathrm{Z}+$ jets then the $t \bar{t}$ productions. The event yields of background and signal models are noted in Table (4) for the dielectron, dimuon and dilepton channels. Similarly, the $p_{t}^{\text {miss }}$ distributions of signal models and SM backgrounds after the final selection, are shown in Figure $(7$ a) for the dielectron, Figure ( $7 \mathrm{~b}$ ) for the dimuon, and Figure (8) for the dilepton. In Table (5), the number of the remaining signals and backgrounds is listed after the final selection. A significant reduction of $\mathrm{Z}+$ jets was observed, in addition to a considerable reduction in $\mathrm{t} \overline{\mathrm{t}}$, diboson and triboson backgrounds. 


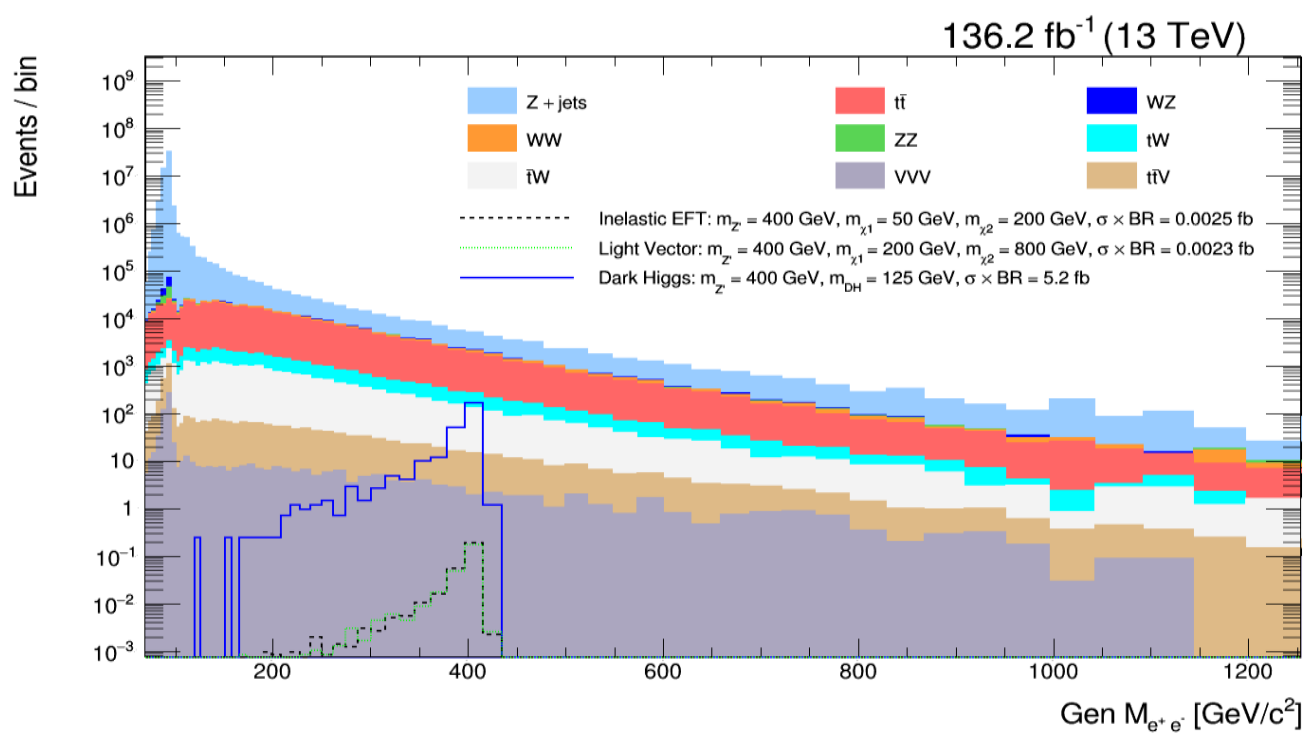

(a)

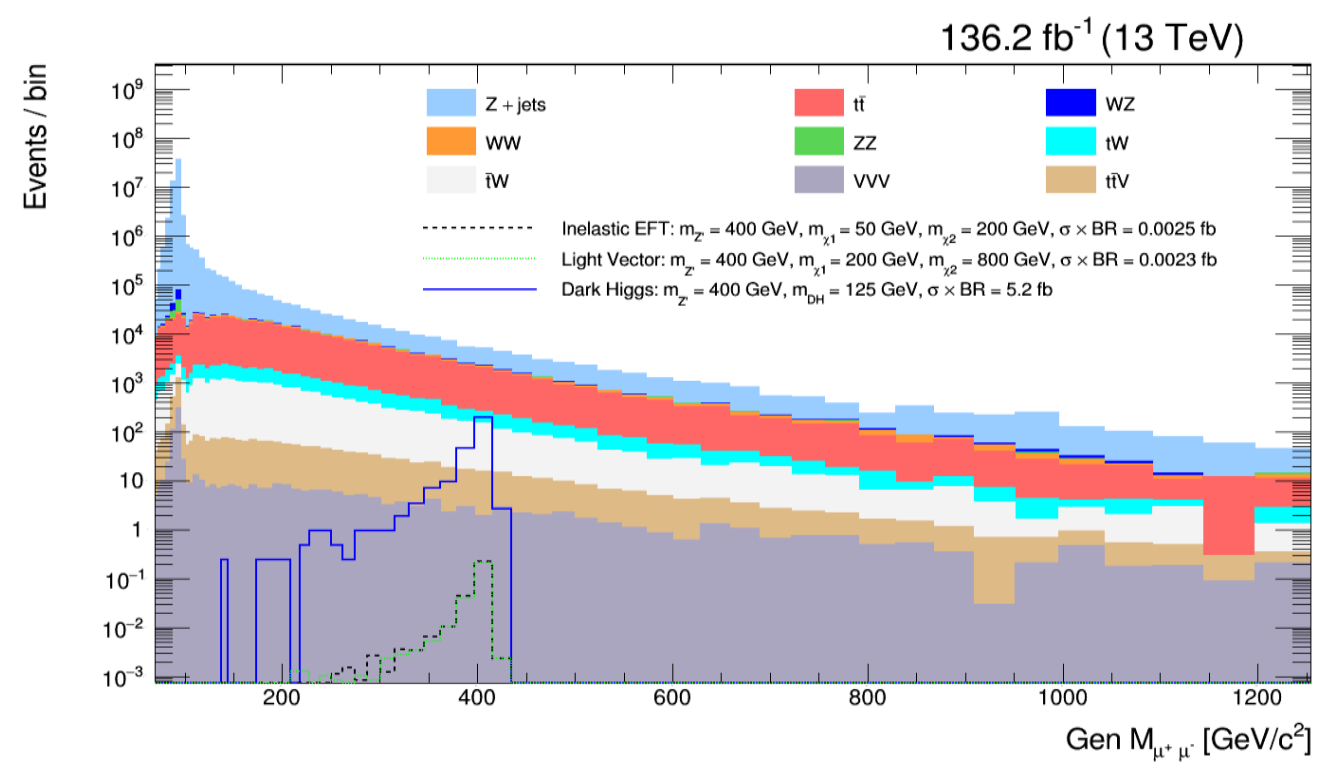

(b)

Fig. (2): The invariant mass distribution at the generator level in cases of dielectron $M_{e+e-}$ (a) and dimuon $M_{u+\mu-}$ (b), after applying the preliminary selection, for the signal models and SM backgrounds. The signals; DHM (Blue solid line), LVM (Green dotted line), and EFT model (Black dashed line) are embedded inside the SM backgrounds (which are presented as colored histograms). 


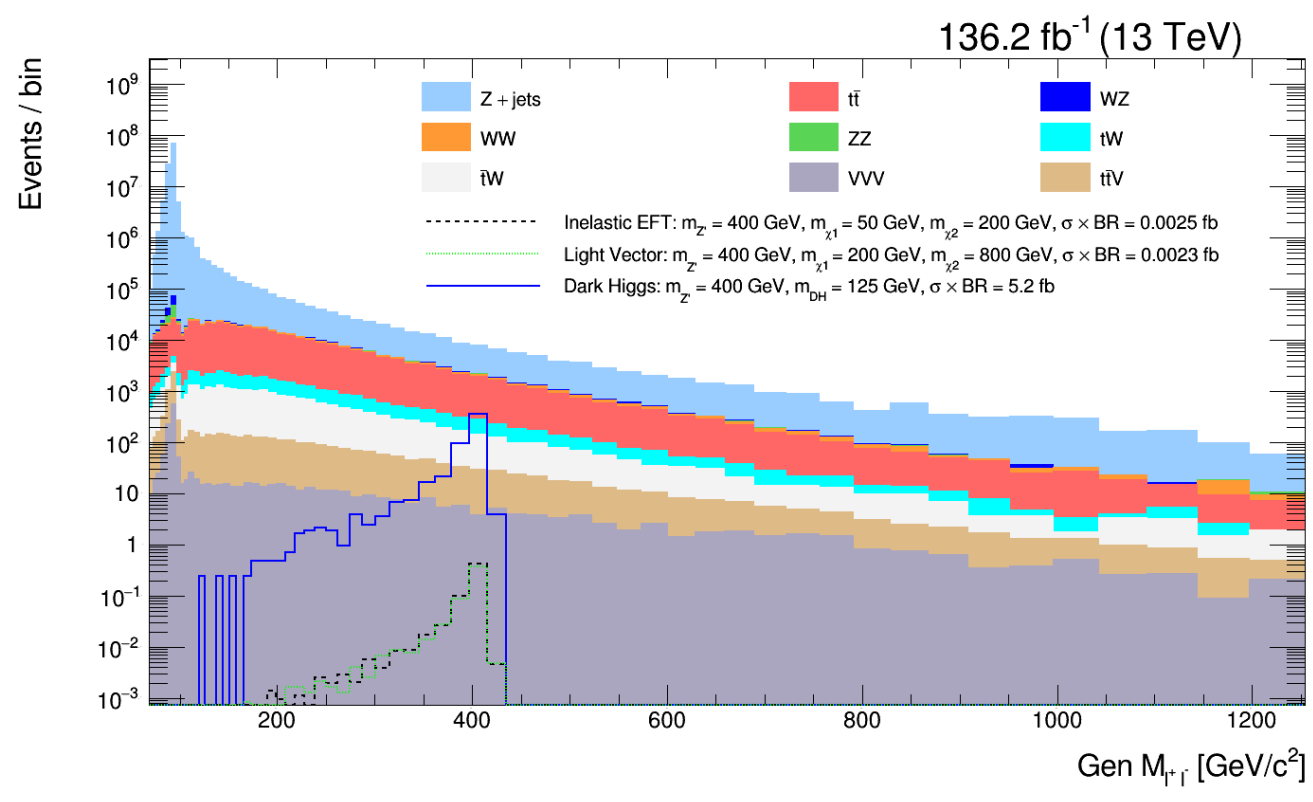

Fig. (3): The invariant mass distribution at the generator level in case of dilepton $M_{l+l^{-}}$, after applying the preliminary selection, for the signal models and SM backgrounds. The signals; DHM (Blue solid line), LVM (Green dotted line), and EFT model (Black dashed line) are embedded inside the SM backgrounds (which are presented as colored histograms).

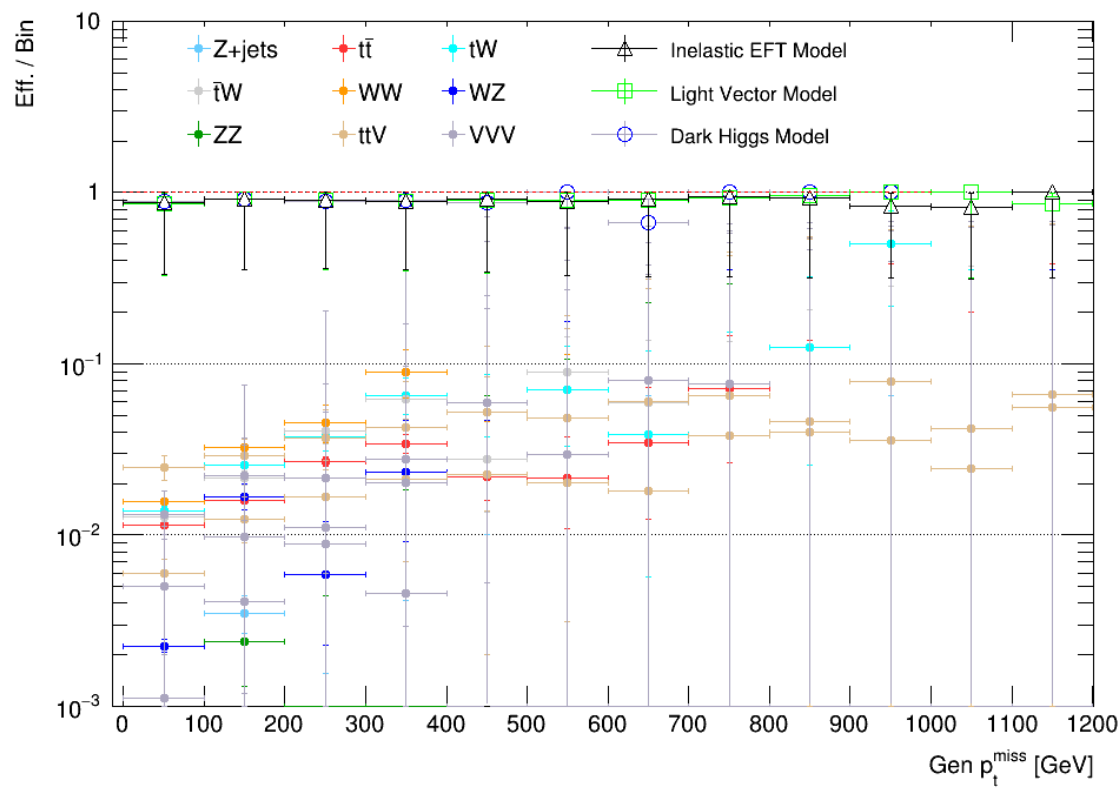

Fig. (4): Efficiency of final selection, computed as explained in text, is plotted versus the generated $p_{t}^{\text {miss }}$ for signals and backgrounds events. The signals are illustrated as blue open circles for DHM, green open boxes for LVM, and black open triangles for EFT model, while the SM backgrounds are presented as colored closed circles. Error bars are statistical only. 
Table (3): The final selection conditions

\begin{tabular}{cc}
\hline Parameter & Cut value \\
\hline$p_{t}^{l}$ & $>35 \mathrm{GeV}$ \\
\hline$\left|\eta_{l}\right|$ & $<2.5$ \\
\hline$p_{t}^{\text {miss }}$ & $>10 \mathrm{GeV}$ \\
\hline$M_{Z}$, & $360-425 \mathrm{GeV}$ \\
\hline
\end{tabular}

Table (4): Number of calculated background and signal events for the dielectron, dimuon and combined dilepton channels after applying the preliminary selection conditions

Errors are statistical only.

\begin{tabular}{cccc}
\hline \multirow{2}{*}{ Background or signal } & \multicolumn{3}{c}{ Number of events } \\
\cline { 2 - 4 } & $2 \mathrm{e}$ & $2 \mu$ & $2 l$ \\
\hline $\mathrm{t} \overline{\mathrm{t}}$ & $533035.8 \pm 730.1$ & $547377.3 \pm 739.9$ & $1080413 \pm 1039$ \\
\hline $\mathrm{tW}$ & $30851.90 \pm 175.6$ & $31638.30 \pm 177.9$ & $62490.20 \pm 250.0$ \\
\hline$\overline{\mathrm{t} W}$ & $31311.80 \pm 176.9$ & $31580.15 \pm 177.7$ & $62891.90 \pm 250.8$ \\
\hline $\mathrm{WW}$ & $44664.20 \pm 211.3$ & $47226.00 \pm 217.3$ & $91890.20 \pm 303.1$ \\
\hline $\mathrm{WZ}$ & $58873.80 \pm 242.6$ & $60930.90 \pm 246.8$ & $119804.7 \pm 346.1$ \\
\hline $\mathrm{ZZ}$ & $38617.40 \pm 196.5$ & $40796.60 \pm 202.0$ & $79414.00 \pm 281.8$ \\
\hline $\mathrm{ttZ}$ & $2469.800 \pm 49.70$ & $2530.500 \pm 50.30$ & $5000.300 \pm 70.70$ \\
\hline $\mathrm{ttW}$ & $1135.200 \pm 33.70$ & $1151.300 \pm 33.90$ & $2286.400 \pm 47.80$ \\
\hline $\mathrm{WWZ}$ & $468.2000 \pm 21.60$ & $474.3000 \pm 21.80$ & $942.5000 \pm 30.70$ \\
\hline $\mathrm{WZZ}$ & $208.1000 \pm 14.40$ & $216.1000 \pm 14.70$ & $424.2000 \pm 20.60$ \\
\hline $\mathrm{ZZZ}$ & $68.10000 \pm 8.300$ & $69.20000 \pm 8.300$ & $137.3000 \pm 11.70$ \\
\hline $\mathrm{Z}+\mathrm{jets}$ & $57411529 \pm 7577.0$ & $61128086 \pm 7818.4$ & $118539612 \pm$ \\
\hline Total background & $58153233 \pm 7625.8$ & $61892076 \pm 7867.1$ & $120045307 \pm$ \\
\hline $\mathrm{DHM}$ & $265.0 \pm 16.30$ & $274.9 \pm 16.60$ & $539.9 \pm 23.20$ \\
\hline $\mathrm{LVM}$ & $0.300 \pm 0.500$ & $0.2389 \pm 0.489$ & $0.560 \pm 0.750$ \\
\hline EFT & $0.300 \pm 0.550$ & $0.3100 \pm 0.560$ & $0.610 \pm 0.780$ \\
\hline
\end{tabular}




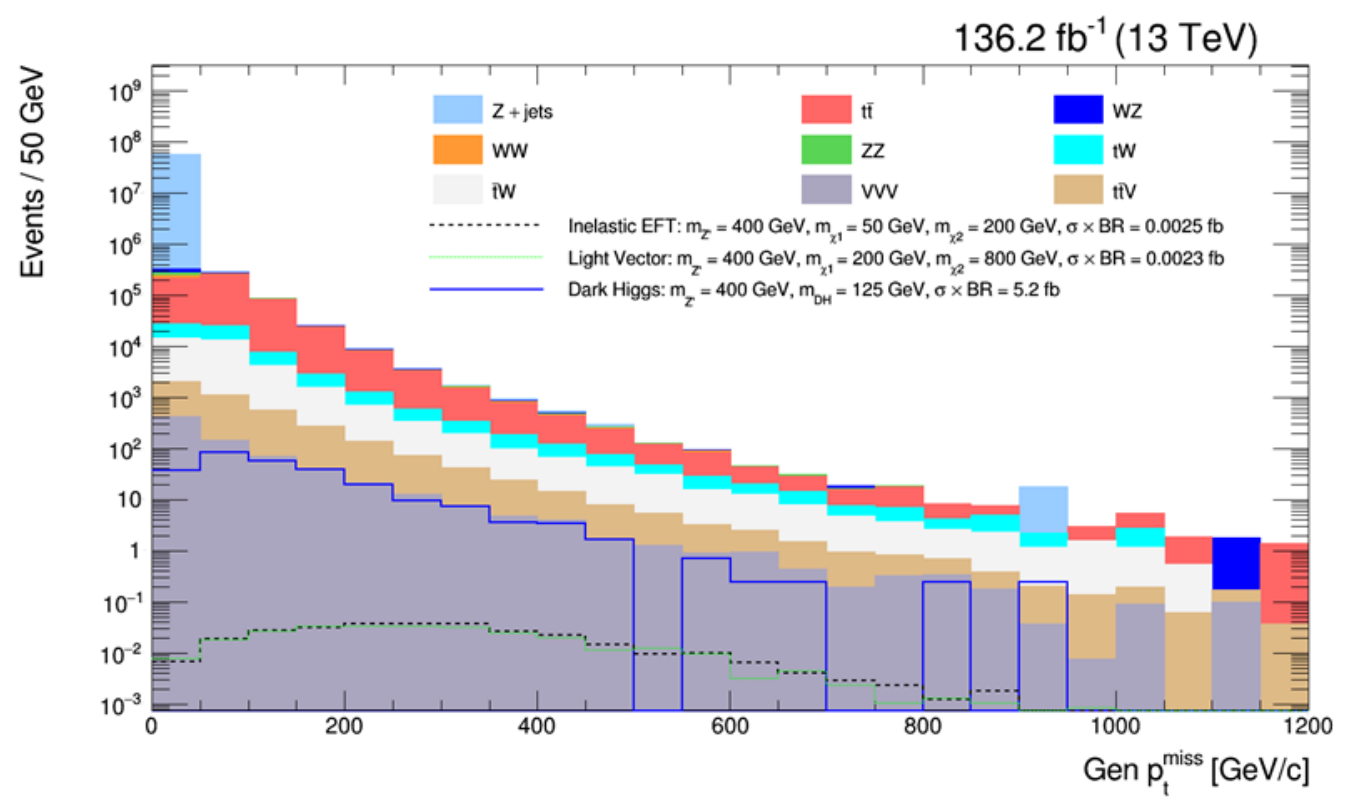

(a)

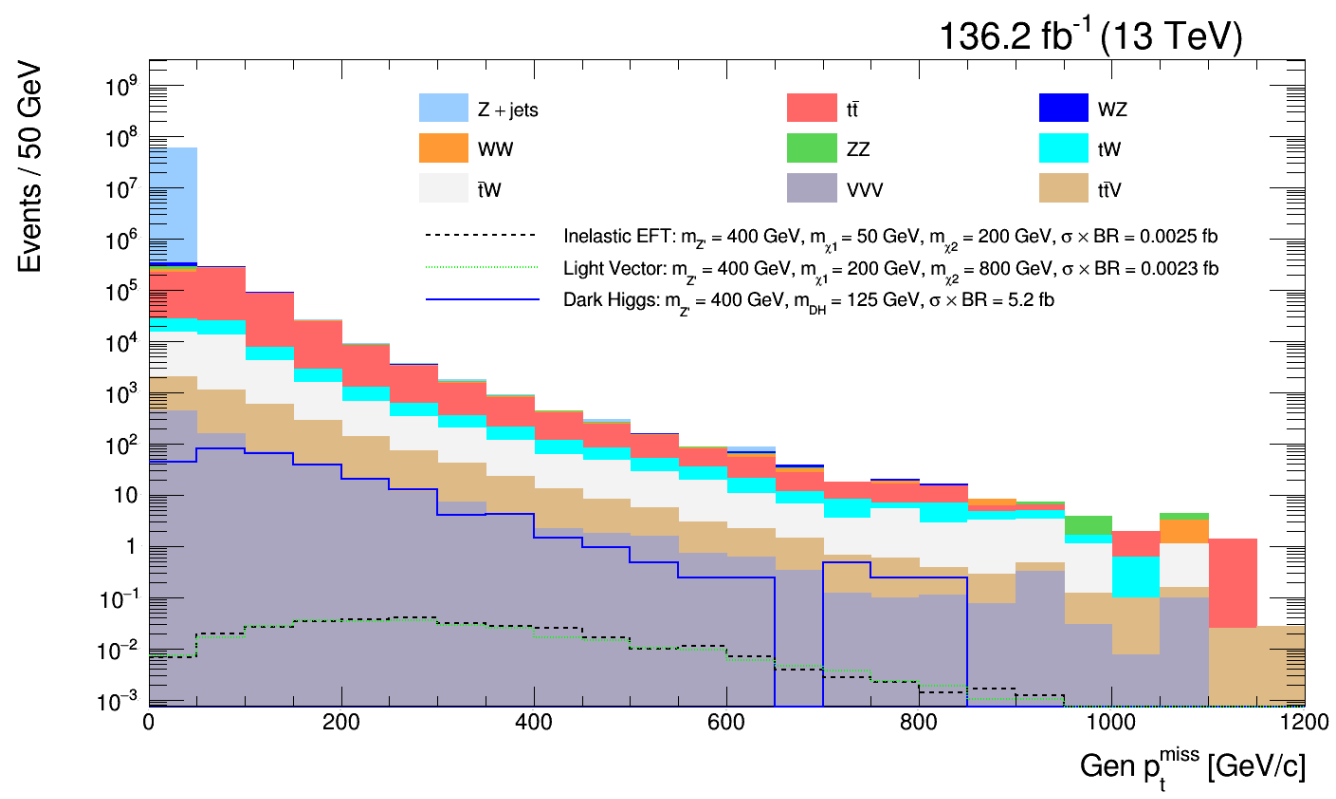

(b)

Fig. (5): The distribution of the missing transverse momentum $p_{t}^{\text {miss }}$, at the generator level in cases of dielectron (a) and dimuon (b); after applying the preliminary selection 


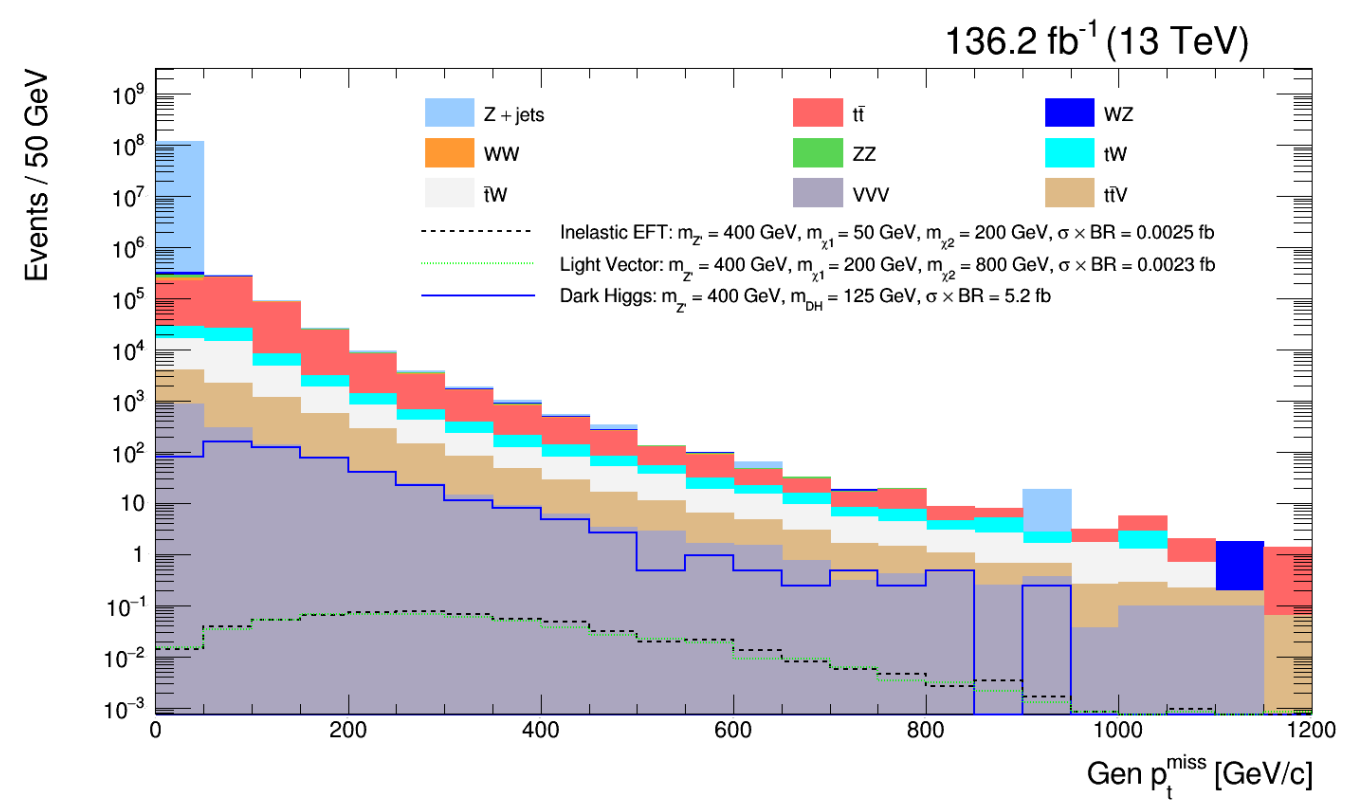

Fig. (6): The distribution of the missing transverse momentum $p_{t}^{\text {miss }}$, at the generator level in case of dileptons; after applying the preliminary selection step

Table (5): Number of calculated background and signal events for the dielectron, dimuon and combined dilepton channels after applying the final selection conditions

Errors are statistical only.

\begin{tabular}{cccc}
\hline \multirow{2}{*}{ Background or signal } & \multicolumn{3}{c}{ Number of events } \\
\cline { 2 - 4 } & $2 \mathrm{e}$ & $2 \mu$ & $2 l$ \\
\hline $\mathrm{t} \overline{\mathrm{t}}$ & $6741.10 \pm 82.1$ & $7122.20 \pm 84.4$ & $13863.3 \pm 118$ \\
\hline $\mathrm{tW}$ & $524.900 \pm 22.9$ & $478.800 \pm 21.9$ & $1003.70 \pm 31.7$ \\
\hline$\overline{\mathrm{tW}}$ & $481.000 \pm 21.9$ & $501.200 \pm 22.4$ & $982.200 \pm 31.3$ \\
\hline $\mathrm{WW}$ & $756.100 \pm 27.5$ & $756.100 \pm 27.5$ & $1512.20 \pm 38.9$ \\
\hline $\mathrm{WZ}$ & $163.400 \pm 12.8$ & $186.300 \pm 13.7$ & $349.700 \pm 18.7$ \\
\hline $\mathrm{ZZ}$ & $6.90000 \pm 2.6$ & $11.5000 \pm 3.40$ & $18.5000 \pm 4.30$ \\
\hline $\mathrm{ttZ}$ & $19.1000 \pm 4.4$ & $19.9000 \pm 4.50$ & $39.0000 \pm 6.30$ \\
\hline $\mathrm{ttW}$ & $31.2000 \pm 5.6$ & $31.8000 \pm 5.60$ & $62.9000 \pm 7.90$ \\
\hline $\mathrm{WWZ}$ & $7.70000 \pm 2.8$ & $7.00000 \pm 2.70$ & $14.8000 \pm 3.80$ \\
\hline $\mathrm{WZZ}$ & $1.20000 \pm 1.0$ & $1.50000 \pm 1.20$ & $2.60000 \pm 1.60$ \\
\hline $\mathrm{ZZZ}$ & $0.10000 \pm 0.4$ & $0.10000 \pm 0.30$ & $0.20000 \pm 0.50$ \\
\hline $\mathrm{Z}+\mathrm{jets}$ & $257.500 \pm 16.0$ & $241.400 \pm 15.5$ & $498.900 \pm 22.3$ \\
\hline Total background & $8990.20 \pm 94.8$ & $9357.90 \pm 96.7$ & $18348.1 \pm 135.5$ \\
\hline $\mathrm{DHM}$ & $230.8 \pm 15.2$ & $254.7 \pm 15.9$ & $485.5 \pm 22.0$ \\
\hline $\mathrm{LVM}$ & $0.240 \pm 0.49$ & $0.270 \pm 0.51$ & $0.500 \pm 0.71$ \\
\hline EFT & $0.260 \pm 0.51$ & $0.290 \pm 0.54$ & $0.550 \pm 0.74$ \\
\hline
\end{tabular}




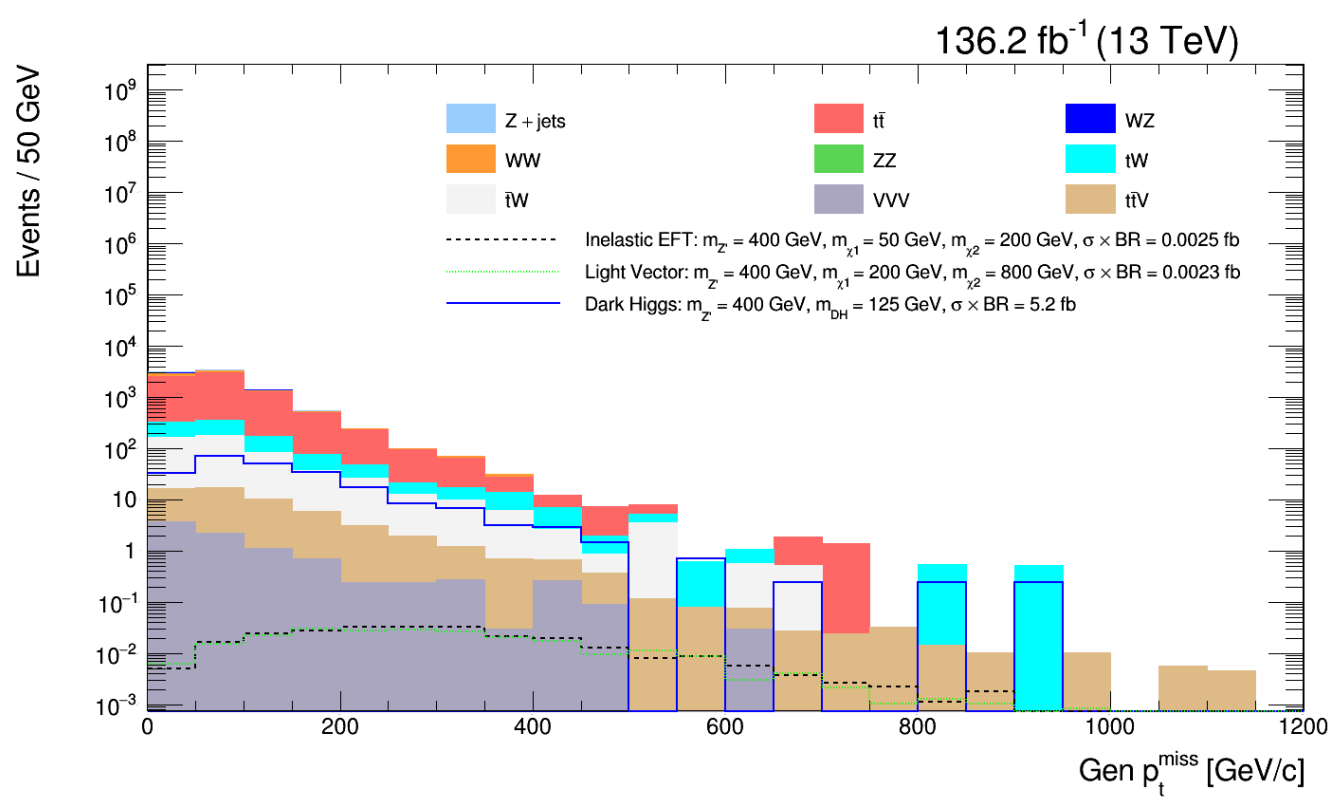

(a)

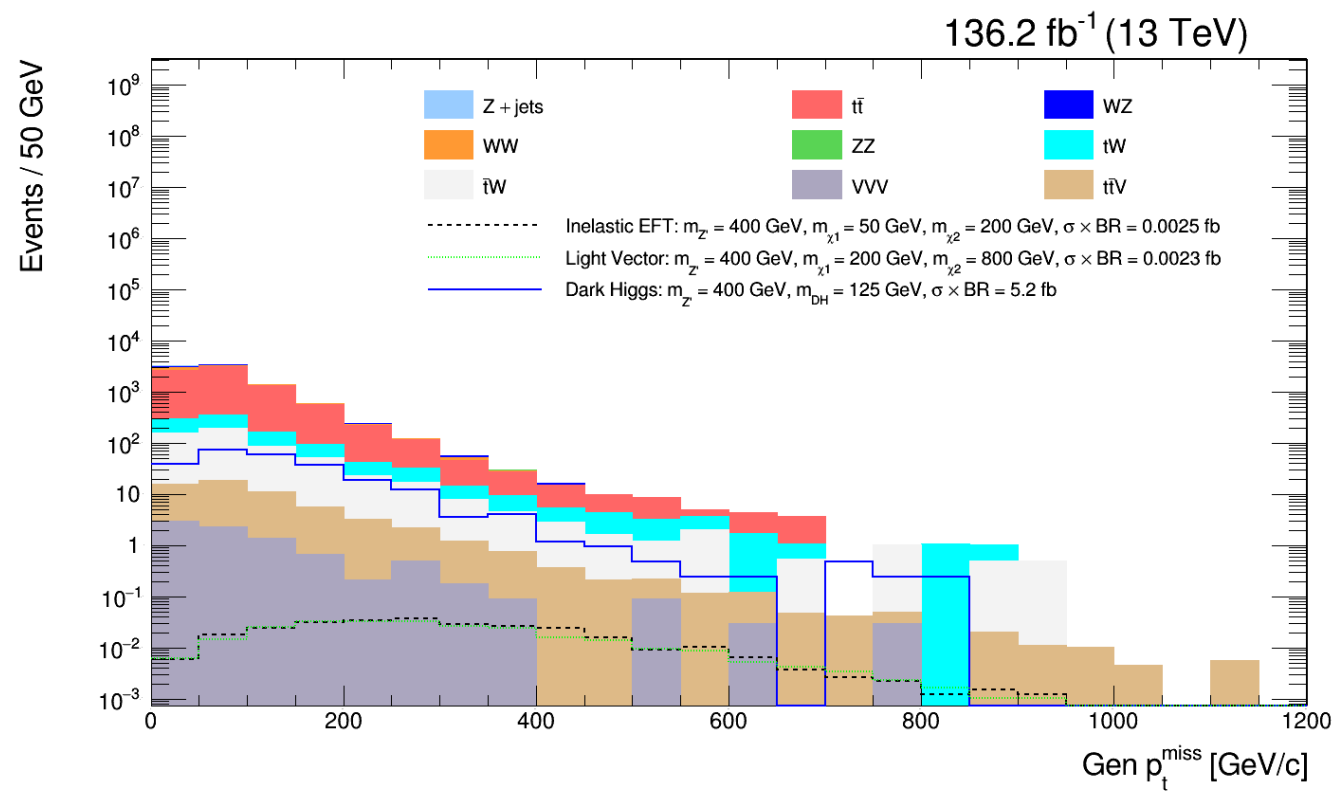

(b)

Fig. (7): The distribution of the missing transverse momentum $p_{t}^{\text {miss }}$, at the generator level in cases of dielectron (a) and dimuon (b); after applying the final selection 


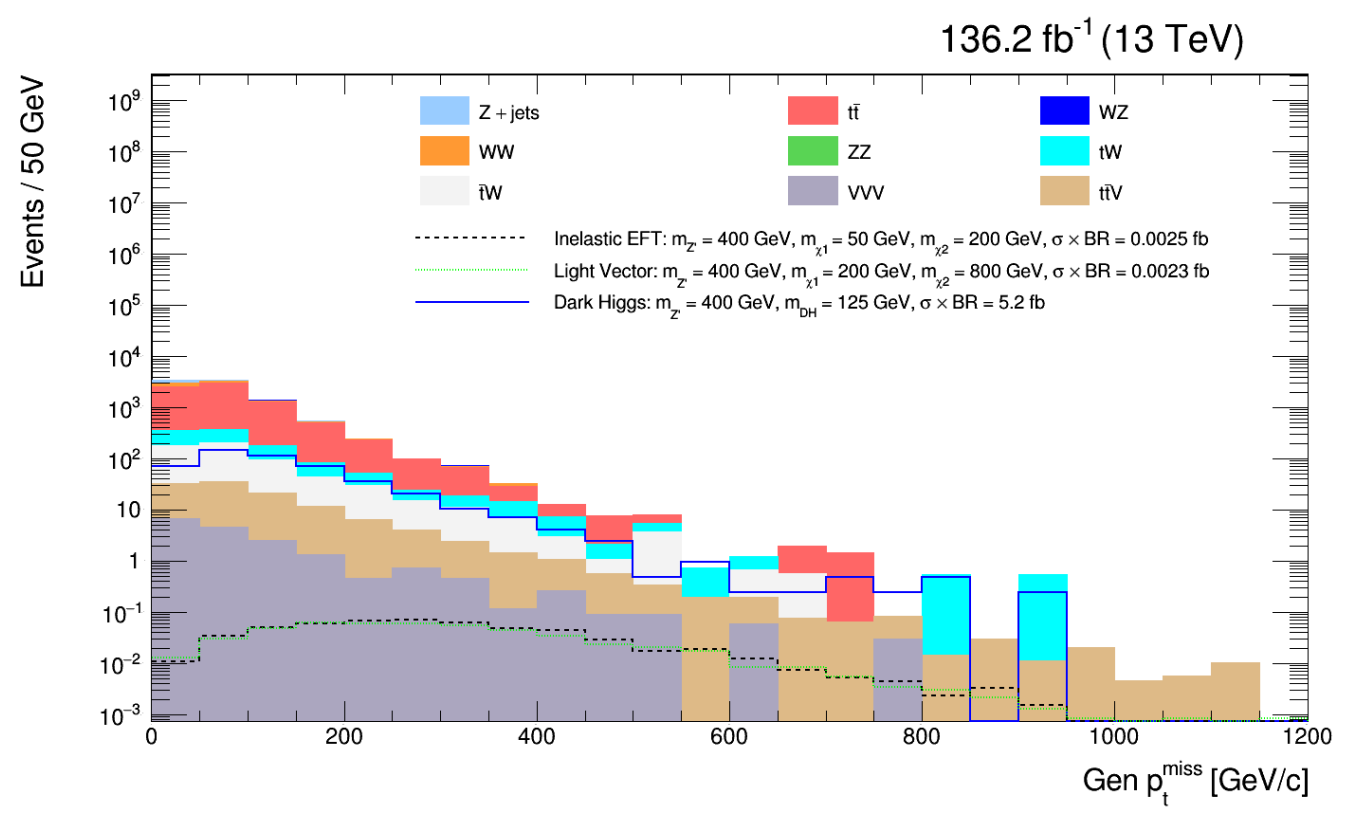

Fig. (8): The distribution of the missing transverse momentum $p_{t}^{\text {miss }}$, at the generator level in cases of dilepton; after applying the final selection

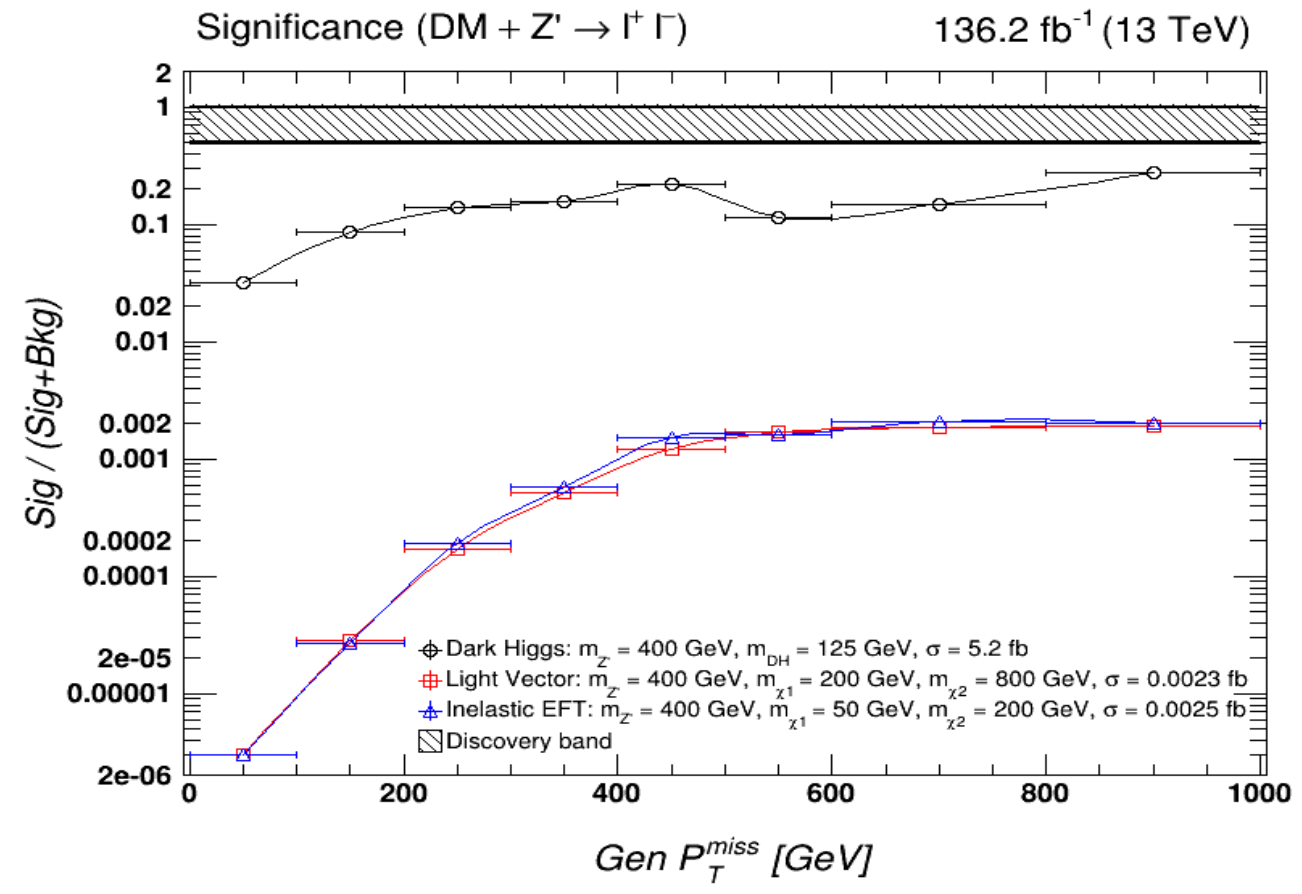

Fig. (9): The signal significance of the models considered., DHM in circles, LVM in boxes and EFT in triangles forms, are presented as a function of bins ((signal / (signal+backgrounds)), in bins: $[0,100],[100,200],[200,300],[300$, $400],[400,500],[500,600],[600,800]$ and $[800,1000] \mathrm{GeV})$, in $p_{t}^{\text {miss }}$ after the final selection. 


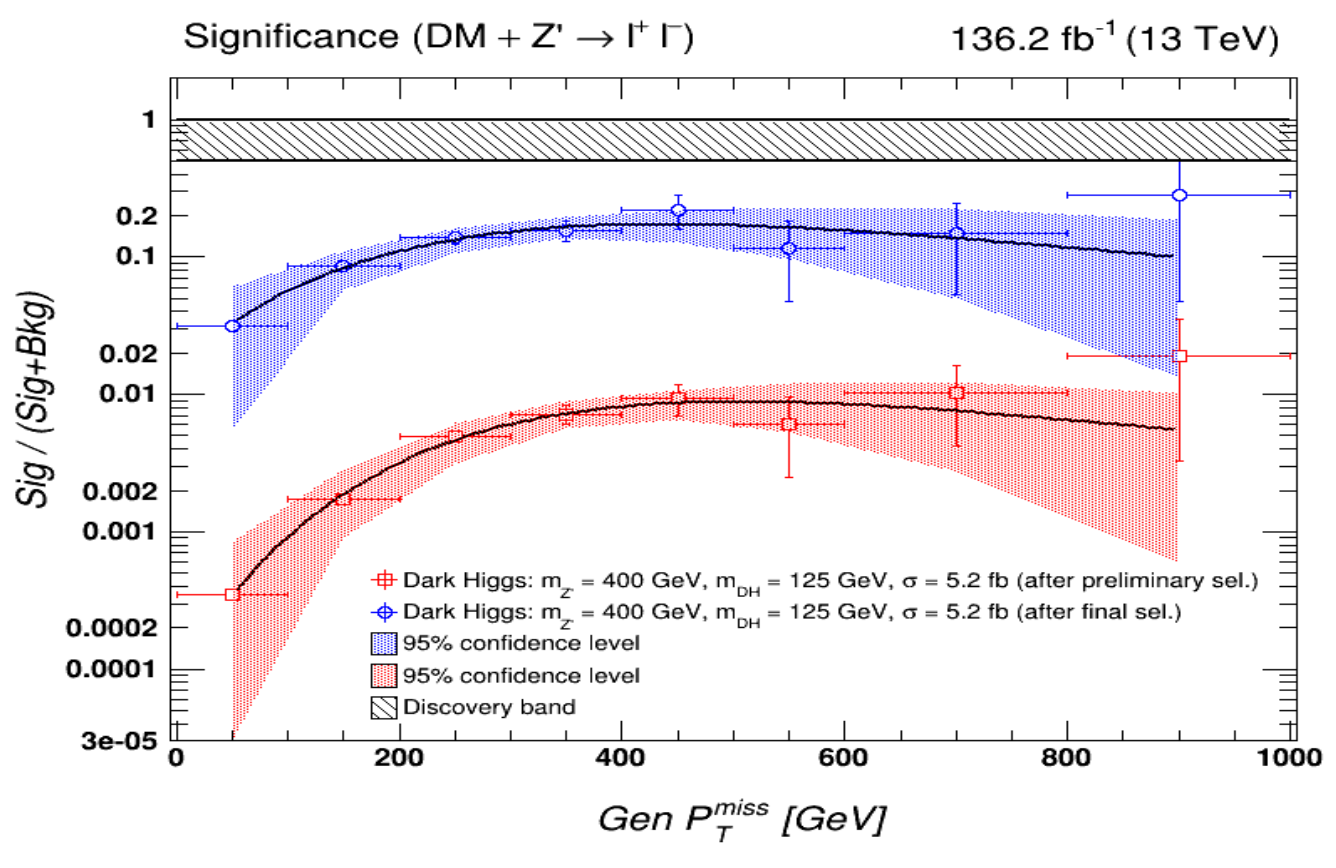

Fig. (10): The significance of the DHM model only before and after the final events selection The vertical error bars are statistical only.

For the purpose of the search for the three signal models DHM, LVM and EFT at the LHC with RUN II requirements (13 TeV center-of-mass energy and integrated luminosity $=136.2 \mathrm{f} \mathrm{b}^{-1}$ ); the significance of each of these models was calculated, which is defined as the number of signal events divided by the number of signals plus the number of backgrounds for each bin, i.e. (signal / (signal+backgrounds)), in bins in $p_{t}^{\text {miss }}$ : [0, 100], [100, 200], [200, 300], [300, 400], [400,500], $[500,600],[600,800]$ and $[800,1000] \mathrm{GeV}$. According to the definition of the significance, the signal events can be greater than the sum of the SM backgrounds events if signal / (signal+backgrounds) $>0.5$, and the significance measurement cannot be greater than 1.0. In Figure (9), the significance of these models, DHM in circles, LVM in boxes and EFT in triangles forms, are presented as a functions of bins in $p_{t}^{\text {miss }}$ after the final selection. In general; the significance for the three models increases at the high values of the $p_{t}^{\text {miss }}$ (above $400 \mathrm{GeV}$ ), among these three signal models the DH is a model with the highest significance, while the other two models (LVM and EFT) almost have the same significance. None of these three models has a number of events exceeds the sum of the SM backgrounds events (i. e. $0.5 \leq \operatorname{signal} /($ signal + backgrounds $) \leq 1.0$, which is represented by the dashed black region "discovery region".

In Figure (9), the significance of DHM versus $p_{t}^{\text {miss }}$ before (in open boxes) and after (in open circles) the final events selection respectively, each of these graphs is fitted using landau function. The fitted functions describe well the significance points (open boxes or open circles), before and after the final events selection, within 95\% confidence level, (these statistical calculations were done using ROOT analysis framework) [16], which insure the goodness of the fit and the significance of the calculations. Figure (10) shows that the significance is improved by about two order of magnitudes after the final events selection in the case of DHM.

\section{Conclusion}

This study presents a search for the dark matter in association with the Z' which is a heavy gauge boson. This work explored three new models, which are Dark Higgs Model; with $\mathrm{M}_{\mathrm{hD}}=125 \mathrm{GeV}$, $\sigma=5.2 \mathrm{fb}$, Light Vector Model, with $\mathrm{M}_{\chi 1}=200$ $\mathrm{GeV}, \mathrm{M}_{\chi_{2}}=800 \mathrm{GeV}, \sigma=0.0023 \mathrm{fb}$, and finally Inelastic EFT; with $\mathrm{M}_{\chi 1}=50 \mathrm{GeV}, \mathrm{M}_{\chi 2}=200 \mathrm{GeV}$, $\sigma=0.0025 \mathrm{fb}$; and $\mathrm{M}_{\mathrm{Z}}=400 \mathrm{GeV}$ for the three models. All of them assume production of new dark sector states and dileptons from Z' decay, and these dark sector states are heavy enough to 
present a candidate for a dark matter. The search was performed using Monte Carlo simulation technique for models and backgrounds, of protonproton collisions at the Large Hadron Collider, with the RUN II requirements (center-of-mass energy of $13 \mathrm{TeV}$ and total integrated luminosity of $136.2 \mathrm{fb}^{-1}$ ).

The experimental signature considered is a large missing transverse momentum accounting for the dark matter candidate plus a pair of opposite-sign leptons from the $Z$ ' leptonic decay. This channel was chosen due to the strong coupling of the $Z$ ' to leptons. The chosen mass of $Z^{\prime}$ is $400 \mathrm{GeV}$, and the pair of leptons were selected through two steps (preliminary and final selections).This was done in order to reduce the SM backgrounds around the $\mathrm{M}_{Z}$, peak. According to the significance test performed in this analysis; it has been found that after the current final events selection, the Dark Higgs Model among the three models under exploration gave the highest significance during the search. In addition, the significance is improved by about two order of magnitudes, within $95 \%$ confidence level, after the final events selection in the case of Dark Higgs model. Other modifications in the models parameters are needed to reach higher cross sections to produce more number of events for the signals with respect to backgrounds. In addition, adding more cuts to the list of cuts in event selection is highly recommended to reduce the backgrounds contamination in the signals regions.

\section{Acknowledgements}

The authors of the present investigation sincerely acknowledge Autran et al. [13], especially Tongyan Lin, for the helpful and useful discussions about their models, and for their support in sending the needed Madgragh the Les Houches Event file format (LHE) for these models. The work presented in this paper was partially financed by the Center for Theoretical Physics (CTP) at British University in Egypt. The work is also supported by the INFN-ASRT joined project, since most of the events have been generated and analyzed, for signals and backgrounds, via the workstation funded by INFN-ASRT joined budget.

\section{References}

1. Planck Collaboration, "Planck 2016 results. XIII. Cosmological parameters”, Astron.Astrophys.,594(2016)A13, doi:10.1051/00046361/201525830.

2. G. Bertone, D. Hooper, and J. Silk, "Particle dark matter: Evidence, candidates and constraints", Phys. Rept. 405 (2005) 279, doi:10.1016/j.physrep.2004.08.031.

3. R. J. Scherrer and M. S. Turner, "On the relic, cosmic abundance of stable, weakly interacting massive doi:10.1103/PhysRevD.33.1585.

4. F. Halzen and A. D. Martin, (1984), "Quarks And Leptons: An Introductory Course In Modern Particle Physics." isbn: 0471887412, 9780471887416.

5. P. Langacker, "The standard model and beyond”, (2010). isbn: 9781420079067.

6. M. Beltran et al., "Maverick dark matter at colliders", doi:10.1007/JHEP09(2010)037.

7. CMS collaboration, V. Khachatryan et al. , (2014), 1410.8812.

8. Y. Bai and T. M. Tait, (2012), 1208.4361.

9. L. M. Carpenter, A. Nelson, C. Shimmin, T. M. Tait, and D. Whiteson, Phys.Rev.D87, 074005 (2013), 1212.3352.

10. L. Carpenter, A. DiFranzo, M. Mulhearn, C. Shimmin, S. Tulin, et al., Phys.Rev.D89, 075017 (2014),1312.2592.

11. M. Beltran, D. Hooper, E. W. Kolb, Z. A. Krusberg, and T. M. Tait, JHEP 1009, 037 (2010), 1002.4137.

12. P. Langacker, Rev.Mod.Phys. 81, 1199 (2009),0801.1345.

13. M. Autran, K. Bauer, T. Lin, and D. Whiteson, ' 'mono- $Z$ ' : searches for dark matter in events with a resonance and missing transverse energy", arXiv:1504.01386v1

14. J. Alwall et al., "The automated computation of tree-level and next-to-leading order differential cross sections, and their matching to parton shower simulations", JHEP07 (2014) 079, doi:10.1007/JHEP07(2014)079.

15. [T. Sj ostrand et al., "An introduction to PYTHIA 8.2", Comput. Phys. Commun.191 (2015) 159, doi:10.1016/j.cpc.2015.01.024.

16. I. Antcheva, M. Ballintijn, B. Bellenot," ROOT - A C + + Framework for Petabyte Data Storage, Statistical Analysis and Visualization, arXiv:1508.07749. 